\title{
New stable QTLs for berry weight do not colocalize with QTLs for seed traits in cultivated grapevine (Vitis vinifera L.)
}

Agnès Doligez , Yves Bertrand, Marc Farnos, Michel Grolier, Charles Romieu, Florence Esnault, Sonia Dias, Gilles Berger, Pierre François, Thierry Pons, Patrick Ortigosa, Catherine Roux, Cléa Houel, Valérie Laucou, Roberto Bacilieri, Jean-Pierre Péros and Patrice This

\begin{abstract}
Background: In grapevine, as in other fruit crops, fruit size and seed content are key components of yield and quality; however, very few Quantitative Trait Loci (QTLs) for berry weight and seed content (number, weight, and dry matter percentage) have been discovered so far. To identify new stable QTLs for marker-assisted selection and candidate gene identification, we performed simultaneous QTL detection in four mapping populations (seeded or seedless) with various genetic backgrounds.

Results: For berry weight, we identified five new QTLS, on linkage groups (LGS) 1, 8, 11, 17 and 18, in addition to the known major QTL on LG 18. The QTL with the largest effect explained up to 31\% of total variance and was found in two genetically distant populations on LG 17, where it colocalized with a published putative domestication locus. For seed traits, besides the major QTLs on LG 18 previously reported, we found four new QTLs explaining up to 51\% of total variance, on LGs 4, 5, 12 and 14. The previously published QTL for seed number on LG 2 was found related in fact to sex. We found colocalizations between seed and berry weight QTLs only for the major QTL on LG 18 in a seedless background, and on LGs 1 and 13 in a seeded background. Candidate genes belonging to the cell number regulator CNR or cytochrome P450 families were found under the berry weight QTLs on LGs 1, 8, and 17. The involvement of these gene families in fruit weight was first described in tomato using a QTL-cloning approach. Several other interesting candidate genes related to cell wall modifications, water import, auxin and ethylene signalling, transcription control, or organ identity were also found under berry weight QTLS.
\end{abstract}

Conclusion: We discovered a total of nine new QTLs for berry weight or seed traits in grapevine, thereby increasing more than twofold the number of reliable QTLs for these traits available for marker assisted selection or candidate gene studies. The lack of colocalization between berry and seed QTLs suggests that these traits may be partly dissociated.

Keywords: Berry weight, Candidate gene, Grapevine, Quantitative trait locus, QTL, Seed number, Seed weight, Vitis vinifera

\section{Background}

Fruit size is a major determinant of both yield and quality for many crops with fleshy fruits. A comprehensive understanding of its genetic determinism is crucial to elucidate fruit development mechanisms and facilitate the breeding of new varieties. Although the genetic architecture of fruit size has been investigated in several species (e.g. tomato, peach, grape, apple, cherry, melon,

* Correspondence: doligez@supagro.inra.fr

INRA, UMR AGAP, Batiment 212 place Viala, F-34060 Montpellier Cedex 1, France citrus, papaya, cranberry), genes underlying Quantitative Trait Loci (QTLs) have been so far identified in tomato only. Grapevine appears as a particularly significant model to study fruit development, since it is the only non-climacteric fruit among the few fruit crop species almost fully sequenced $[1,2]$.

Berry size and seed content are essential selection criteria in grape breeding. Berry size and number are major yield components for both table fruit and wine production. However, large berries are desirable only for table 
grape while smaller fruits are preferred for winemaking. In wine cultivars, small berry size is principally searched for to increase skin-to-flesh ratio, thus improving final concentrations of anthocyanins, tannins and aroma compounds in wine. These chemicals are primarily localized in the skin, but seeds are another important source of condensed tannins. Conversely in table cultivars, reduced seed perceptibility and large berries are sought. Therefore, the possible physiological correlation between berry size and seed quantity is a major issue in grape breeding.

Large variation in berry and seed traits has been observed among cultivars of the cultivated grapevine Vitis vinifera L. On average, berry fresh weight at maturity ranges from 0.5 to $11 \mathrm{~g}$ [3], seed number from 0 to 3.6 and seed weight per berry from 0.03 to $0.22 \mathrm{~g}$ [4].

Similarly to other fleshy fruits [5], grapevine pericarp grows from fertilization to maturity according to a double sigmoid curve, with three main development stages [6-8]. Stage I involves both cell division and enlargement through the accumulation into vacuoles of water and organic acids. During stage II, berry growth slows down or stops. At the onset of the following growth period (stage III), referred to as véraison, many rapid physiological changes occur, among which berry softening and skin coloring in red cultivars. Berry size roughly doubles during this ripening phase, as a result of vacuolar expansion triggered by water and sugars accumulation in the mesocarp (flesh). Maximal berry size depends on both cell number and size [5,7] and is largely defined already at véraison [9]. Berry growth is under the control of several growth regulators: ethylene $[10,11]$, auxins and ABA [12], and gibberellins [13,14].

Normal seed development from fertilization to maturity involves the three following phases: i) rapid cell division; ii) reserve accumulation and cell expansion due to water uptake; iii) slowing down and then arrest of reserve accumulation [15]. In the case of stenospermocarpic seedlessness, which originates from cv. Sultanina, intensively used as a genitor in table grape breeding, the ovule is successfully fertilized, embryo and endosperm cell divisions begin, but then the endosperm degenerates at various stages. Seeds do not achieve full development, although embryos are viable and can develop into new plants [16]. Stenospermocarpic seedlessness can therefore be described with several quantitative traits (seed number, fresh and dry weight, seed coat hardness, endosperm development) [17], some of which will also vary in seeded grapes.

The widely accepted correlation between berry weight and seed content is not so evident. A positive correlation between berry final weight and seed content has been frequently observed within seeded cultivars $[8,18-23]$, within populations segregating for seedlessness [24-30] and in pools composed of both seeded and seedless table grape breeding populations [31,32]. By contrast, no significant correlation was found between seed content and berry size within a set of 190 new table grape cultivars [33] or in a set of 254 highly diverse cultivars [4]. It is generally admitted that the correlation between berry size and seed content mainly results from growth regulators produced by the seeds $[16,34,35]$. However, some authors found that exogenous gibberellins had no effect on berry size in seeded cultivars [34,36,37].

Despite high heritabilities, the genetic determinism of berry weight and seed content variation is far less documented than the effect of environmental factors. Heritability of berry weight and seedlessness seems relatively high and mainly additive. Indeed, reported values for narrow-sense heritability were $0.63-0.69$ for berry weight $[31,32,38]$ and 0.58 for seedlessness [31]. Similarly, broadsense heritability values were $0.49-0.92$ for berry weight [39-42], 0.996 for total seed weight per berry [39], and 0.34 for seed number [39]. All previously published QTL studies in a stenospermocarpic seedless background found a major QTL for both berry weight and seed traits on LG 18 [25,27-29]. The most probable candidate gene for this QTL is the VvAGL11 gene, an ortholog of a MADS-box gene involved in ovule differentiation in Arabidopsis and petunia [30]. For berry size, only two QTLs stable over time were found in addition to the above QTL on LG18 [43], the other ones being unstable (Additional file 1: Table S1). For seed traits, four stable minor QTLs were reported in addition to the major QTL on LG 18. Houel et al. $[44,45]$ found five SNPs associated to berry weight. Despite all these efforts, no gene involved in berry size variation has been identified so far.

The objectives of this study were: i) to find QTLs for berry weight and seed traits stable enough to be used in Marker Assisted Selection and for searching for candidate genes harboring causal mutations, ii) to assess the extent of seed and berry QTLs colocalization, and iii) to propose positional candidate genes for further evaluation. To achieve these goals, we performed simultaneous QTL detection over several years in various genetic backgrounds (two seedless and two seeded pseudo-F1 families). This allowed us to discover five new stable QTLs for berry weight and four new stable QTLs for seed traits, with little colocalization between berry and seed QTLs, and to propose a few promising candidate genes in these regions.

\section{Methods}

\section{Plant material}

This study was based on four pseudo-F1 mapping populations (Table 1). MTP3140 and MTP3234 populations were 
Table 1 Progenies and experimental designs used for QTL detection in four grapevine mapping populations

\begin{tabular}{|c|c|c|c|c|c|c|c|c|}
\hline Population & Parents & $\begin{array}{l}\text { Nb. } \\
\text { offsprings }\end{array}$ & $\begin{array}{l}\text { Genetic } \\
\text { background }\end{array}$ & $\begin{array}{l}\mathrm{Nb} . \\
\text { replicates }\end{array}$ & $\begin{array}{l}\text { Nb. plants / } \\
\text { offspring }\end{array}$ & $\begin{array}{l}\text { Years of harvest } \\
\text { and phenotyping }\end{array}$ & $\begin{array}{l}\text { Nb.clusters } \\
\text { harvested per } \\
\text { offspring }\end{array}$ & $\begin{array}{l}\text { Reference of } \\
\text { genetic map }\end{array}$ \\
\hline \multirow[t]{2}{*}{ MTP3140 } & $\begin{array}{l}\text { MTP2223-27 (Dattier de } \\
\text { Beyrouth } \times 75 \text { Pirovano) }\end{array}$ & 139 & \multirow[t]{2}{*}{$\begin{array}{l}\text { table } \\
\text { seedless }\end{array}$} & \multirow[t]{2}{*}{1} & \multirow[t]{2}{*}{1} & \multirow[t]{2}{*}{$\begin{array}{l}1994^{4}, 1995^{4}, 1996^{4}, \\
1998,1999\end{array}$} & \multirow[t]{2}{*}{ all } & \multirow[t]{2}{*}[48]{} \\
\hline & $\begin{array}{l}\text { x MTP2121-30 (Alphonse } \\
\text { Lavallée } \times \text { Sultanine) }\end{array}$ & & & & & & & \\
\hline \multirow[t]{2}{*}{ MTP3234 } & $\begin{array}{l}\text { MTP2687-85 (Olivette } \\
\text { noire } \times \text { Ribol) }\end{array}$ & \multirow[t]{2}{*}{174} & \multirow[t]{2}{*}{ table seeded } & \multirow[t]{2}{*}{1} & \multirow[t]{2}{*}{1} & \multirow[t]{2}{*}{$2002^{5}, 2003,2004$} & \multirow[t]{2}{*}{ all } & \multirow[t]{2}{*}[47]{} \\
\hline & x Muscat of Hamburg) & & & & & & & \\
\hline \multirow[t]{2}{*}{$S \times G^{1,2}$} & Grenache $\times$ Syrah & 96 & \multirow[t]{2}{*}{ wine seeded } & \multirow[t]{2}{*}{2} & \multirow[t]{2}{*}{5} & \multirow[t]{2}{*}{$2005^{6}, 2006,2007$} & \multirow[t]{2}{*}{8} & \multirow{2}{*}{$\begin{array}{l}\text { modified } \\
\text { from }[49]^{8}\end{array}$} \\
\hline & Syrah $\times$ Grenache & 95 & & & & & & \\
\hline \multirow[t]{2}{*}{ MTP3346 3} & Muscat of Alexandria & 519 & \multirow{2}{*}{$\begin{array}{l}\text { table } \\
\text { seedless }\end{array}$} & \multirow[t]{2}{*}{1} & \multirow[t]{2}{*}{1} & \multirow[t]{2}{*}{$2003,2005^{7}$} & \multirow[t]{2}{*}{ all } & \multirow[t]{2}{*}[48]{} \\
\hline & $\begin{array}{l}\text { x MTP3140-517 (selected } \\
\text { from the MTP3140 } \\
\text { population) }\end{array}$ & & & & & & & \\
\hline
\end{tabular}

${ }^{1}$ Reciprocal cross.

${ }^{2}$ Seed dry weight not evaluated in SxG.

${ }^{3}$ Partial QTL detection in ten genomic regions only.

${ }^{4}$ Phenotypic data already available from [46] and [25], with only slight differences in phenotyping protocol.

${ }^{5}$ Seed traits not evaluated in 2002.

${ }^{6}$ Phenotyping of one replicate only in 2005.

${ }^{7}$ Phenotyping on a sample of 142 individuals in 2005.

${ }^{8}$ Maps of Huang et al. [49] but based on the Kosambi (instead of Haldane) mapping function.

over-grafted in 1993 [46] and 2001-2002 [47], respectively, at the INRA Chapitre experimental station, Hérault, France. A population obtained from a cross between cv. Syrah and cv. Grenache (SxG) was grafted and planted in two complete randomized blocks in 2003 at the same experimental station. MTP3346 progeny plants were grown in a greenhouse at the CTIFL experimental station of Balandran, Gard, in 20012003. In 2004, a random subset of fertile plants from this population were installed outside in containers at the Chapitre experimental station, without replication.

\section{Phenotyping}

Clusters of each fruit-bearing offspring were harvested at maturity during at least two years (Table 1). End parts of clusters were discarded and 100 berries randomly sampled and weighted to estimate mean berry weight (MBW). Seeds were extracted from 25 random berries to determine mean seed number per berry (MSN), total seed fresh weight per berry (TSFW), mean seed fresh weight (MSFW) and the percentage of seed dry matter after drying at $80^{\circ} \mathrm{C}$ for 72 hours (\%SDM). The residuals obtained by linear regression using SAS/STAT ${ }^{\circ}$ software v9.1.3 (SAS Institute Inc., Cary, NC) of MBW on either MSN or TSFW (RESN and RESFW, respectively) were used as additional traits for QTL analyses.

\section{Normality, genetic values and heritability}

Statistical analyses were performed on raw phenotypic data from each year using the $\mathrm{R}$ statistical base and pgirmess packages [50]. Distribution normality was evaluated using the Shapiro-Wilk test [51]. Since most data distributions significantly deviated from normality, we used nonparametric procedures to calculate and test phenotypic correlations (Spearman rank-order correlation coefficient).

When data distribution deviated from normality, we applied either square root (sqrt) or neperian logarithm (ln) transformation to unskew distribution, after adding 1,2 or 3 to the raw data to obtain positive values. These transformed values were used to estimate the Best Linear Unbiased Predictors (BLUP) of genetic values across blocks and/or years with SAS/STAT, for use in QTL detection. Models selected were those with the lowest Bayesian Information Criterion (BIC), among several models always including a random genotypic effect, completed or not by a fixed year and/or block effect. For the SxG population, the full model was: $P_{i j k}=\mu+G_{i}+b_{j}+y_{k}$ $+e_{i j k}$, where $P_{i j k}$ was the phenotypic value of genotype $i$ in block $j$ and year $k, \mu$ the overall mean, $G_{i}$ the random effect of genotype $i, b_{j}$ the fixed effect of block $j, y_{k}$ the fixed effect of year $k$ and $e_{i j k}$ the residual error effect. Genetic correlations (Spearman's test) were estimated between BLUPs. Variance estimates of the selected models were used to estimate broad-sense heritabilities on an interannual genotype mean basis, defined as $H^{2}=\sigma_{\mathrm{G}}^{2} /$ $\left(\sigma_{\mathrm{G}}^{2}+\left(\sigma_{\mathrm{e}}^{2} / n\right)\right)$, where $\sigma_{\mathrm{G}}^{2}$ and $\sigma_{\mathrm{e}}^{2}$ were the genotypic and residual variances, respectively, and $n$ the mean number of replicates ( $n=1$ when year effect was significant, otherwise $n=$ mean number of replicates with non-missing data). 


\section{QTL detection}

Parental and consensus genetic maps were already available (Table 1). Since only a partial genetic map was available for population MTP3346 (10 genomic regions spanning less than a quarter of the genome), the results for this population were provided as additional files and only mentioned to complement the other results.

A summary of map features is given in Additional file 2: Table S2). QTL detection was performed on parental and consensus maps as follows. Composite interval mapping (CIM) was applied to parental maps using WinQTLCart 2.0 [52], with cofactors selected through forward and backward (FB) regressions. LOD thresholds corresponding to a genome-wide type I error rate of $\alpha=5 \%$ were determined using 1,000 permutations of traits over marker data, with cofactors reselected for each permuted data set, using QTLCartographer 1.17 [53,54].

MQM (Multiple QTL Model, a CIM equivalent) was performed on consensus maps using MapQTL 4.0 [55], with cofactors selected as the markers nearest to the QTLs detected with interval mapping (IM) and which passed the MapQTL automatic cofactor selection procedure (backward elimination). The $\alpha=5 \%$ genome-wide LOD thresholds were those obtained for IM with 1,000 permutations, empirically corrected by adding 0.5 to take into account higher threshold values in CIM compared to IM. To check dubious QTLs (e.g. in case of distorted segregation, large interval between nearest markers, or skewed distribution of residuals), the CIM results were complemented by a non-parametric Kruskal-Wallis rank sum test (for the consensus map) or variance analysis (for parental maps). Confidence intervals of QTL positions were defined as one-LOD support intervals. QTL detection was carried out with the transformed phenotypic data for each year (BLUPs of genetic values across blocks for SxG in 2006 and 2007) to check QTL stability over time, but also on the BLUPs of genetic values across years. For QTLs detected on consensus maps, female, male and dominance allelic effects were estimated according to [56], as $A f=[(\mu a d+\mu a c)-(\mu b d+\mu b c)] / 4, A m=[(\mu a c+\mu b c)-(\mu a d+$ $\mu b d)] / 4$ and $D=[(\mu a c+\mu b d)-(\mu b c+\mu a d)] / 4$, respectively, where $\mu a c, \mu a d, \mu b c$ and $\mu b d$ were the phenotypic means estimated for each of the four possible genotypic classes, $a c, a d, b c$ and $b d$, respectively, in the progeny of an $a b \times$ $c d$ cross.

\section{Candidate genes}

To identify the most probable candidate genes, we used a double approach. First, consulting the available literature or databases, we established a list of genes possibly involved in berry weight according to their putative function (e.g. cell replication, water transport, or cell wall metabolism), as well as to the role of their homologue on fruit weight in other species or to published results obtained for expression, transformation or association genetics in grapevine. We then checked whether these candidate genes colocalized with the detected QTLs in our mapping populations. In particular, we explored two gene families involved in fruit weight QTLs cloned in tomato, the CNR and P450 78A families [57,58]. To search for homology and genome position, we used the BlastP algorithm to match the proteic sequence against the NCBI Vitis refseq_protein database.

Secondly, as a complementary approach, we looked for the limited number of genes with a putative function relevant to berry weight, within the numerous genes present under the QTLs, even in the absence of published results in grapevine or cloned QTLs in other fleshy fruit species. Taking into account a review of fine mapped QTLs [59], showing that causal polymorphisms were found within $3 \mathrm{cM}$ of LOD peaks, we extracted positional candidate genes from $\mathrm{a}+/-3 \mathrm{cM}$ interval around the LOD peak of each stable BLUP QTL for berry weight on consensus maps. The physical interval limits were extrapolated from the positions of flanking SSR markers. Accession numbers, positions and putative functions of predicted mRNA were extracted from ref_12X_top_level. gff3.gz, last modification 03/15/2012 downloaded at ftp://ftp.ncbi.nih.gov/genomes/Vitis_vinifera/GFF/, obtained with the GNOMON method (http://www.ncbi.nlm.nih.gov/ genome/guide/gnomon.shtml). We then checked in QTL intervals the presence of functional candidate genes.

\section{Results}

\section{Phenotypic data}

Raw measurement distributions were very similar between years but showed differences between populations (Additional file 3: Figure S1). The extent of variation in berry weight was larger for the three table grape populations than for the SxG wine grape population, whereas it was more similar among populations for seed number, despite the large zero class in the seedless MTP3140 population. Seed weights presented more variation in MTP3140 and MTP3234 than in SxG and MTP3346.

All traits showed continuous variation and transgressive segregation. Regression of MBW on MSN or TSFW was always highly significant, except for MSN in SxG in 2006 and 2007. Deviation from normality was observed for most traits in most populations and appropriate transformations were therefore applied (Additional file 4: Table S3). Phenotypic correlation between years varied from 0.38 to 0.97 depending on trait and population (Additional file 5: Table S4) and was always highly significant.

Most of the models selected to estimate BLUPs of genetic values and heritabilities included year effect (Additional file 4: Table S3). In SxG population, selected models never 
included block effect (for both within and among year models). Genetic correlation between traits are shown in Figure 1 and Additional file 6: Figure S2). Berry weight was highly significantly correlated with total seed fresh weight in all populations $(P<0.001)$, the correlation value varying between 0.29 and 0.80 . Correlation values between berry weight and seed number were lower (0.10-0.51) and not always significant $(P>0.05$ for SxG). RESN and RESFW were actually uncorrelated to seed number and total fresh weight, as expected, whereas they remained tightly correlated with berry weight (0.59-0.99) and between each other (0.77-0.96). The correlation between seed number and mean seed weight was significantly positive in the two seedless populations but not significant or significantly negative in the two seeded populations.

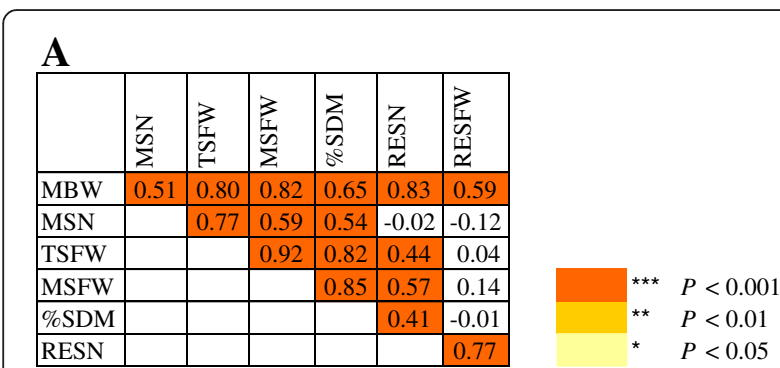

\section{B}

\begin{tabular}{|c|c|c|c|c|c|c|}
\hline & $z_{z}^{Z}$ & 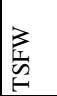 & $\frac{3}{\mathbf{3}}$ & $\begin{array}{l}\sum_{0} \\
\text { 20 }\end{array}$ & $\begin{array}{l}z \\
z \\
1 \\
\underline{1}\end{array}$ & 敨 \\
\hline MBW & 0.33 & \begin{tabular}{|l|l} 
\\
\end{tabular} & 0.39 & 0.04 & \begin{tabular}{|l|}
0.89 \\
\end{tabular} & 0.84 \\
\hline MSN & & \begin{tabular}{|l|l|} 
& 0.83 \\
\end{tabular} & 0.04 & 0.32 & \begin{tabular}{|l|}
0.03 \\
\end{tabular} & \begin{tabular}{|l|}
-0.02 \\
\end{tabular} \\
\hline TSFW & & & 0.52 & 0.25 & \begin{tabular}{|l|}
0.27 \\
\end{tabular} & \begin{tabular}{|l|}
0.09 \\
\end{tabular} \\
\hline MSFW & & & & 0.09 & \begin{tabular}{|l|}
0.43 \\
\end{tabular} & \begin{tabular}{|l|}
0.18 \\
\end{tabular} \\
\hline$\%$ SDM & & & & & \begin{tabular}{|l|}
-0.06 \\
\end{tabular} & \begin{tabular}{|l|l|}
-0.07 \\
\end{tabular} \\
\hline RESN & & & & & & 0.95 \\
\hline
\end{tabular}

C

\begin{tabular}{|c|c|c|c|c|c|c|}
\hline & $\mid \begin{array}{l}Z \\
n \\
n\end{array}$ & 㦂 & 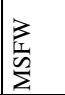 & $\begin{array}{l}5 \\
0 \\
0 \\
5\end{array}$ & 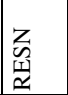 & 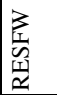 \\
\hline MBW & \begin{tabular}{|l|}
0.10 \\
\end{tabular} & 0.33 & 0.21 & - & 0.99 & 0.95 \\
\hline \begin{tabular}{|l|} 
MSN \\
\end{tabular} & & 0.61 & \begin{tabular}{|c|}
-0.56 \\
\end{tabular} & - & \begin{tabular}{|l|}
0.02 \\
\end{tabular} & -0.08 \\
\hline \begin{tabular}{|l} 
TSFW \\
\end{tabular} & & & 0.27 & - & \begin{tabular}{|l|}
0.29 \\
\end{tabular} & 0.07 \\
\hline \begin{tabular}{|l} 
MSFW \\
\end{tabular} & & & & - & \begin{tabular}{|l|}
0.27 \\
\end{tabular} & 0.16 \\
\hline$\% \mathrm{SDM}$ & & & & & - & - \\
\hline RESN & & & & & & \begin{tabular}{|l|l|}
0.96 \\
\end{tabular} \\
\hline
\end{tabular}

Figure 1 Genetic correlations between seven seed and berryrelated traits within years in three grapevine mapping populations. Genetic correlations among BLUPs of the genetic value for the populations MTP3140 (A), MTP3234 (B), SxG (C). Background cell color indicates Spearman test significance: $a=5 \%$ (light yellow), 1\% (dark yellow), $0.1 \%$ (orange), not significant (white). MBW: mean berry weight; MSN: mean seed number; TSFW: total seed fresh weight; MSFW: mean seed fresh weight; \%SDM: seed dry matter percentage; RESN: residual berry weight unexplained by seed number; RESFW: residual berry weight unexplained by total seed fresh weight.
Broad sense heritability of the interannual genotypic means was rather large and varied between 0.51 and 0.95 (Table 2 and Additional file 7: Table S5).

\section{QTL analysis}

QTLs detected from consensus and parental maps are listed in Tables 3 and 4, Additional file 8: Table S6 and Additional file 9: Table S7 (main features of stable QTLs only), and Additional file 10: Table S8 and Additional file 11: Table S9 (detailed features of all QTLs). Confidence intervals are shown on Figure 2 and Additional file 12: Figure S3 and Additional file 13: Figure S4. In the following, we will focus on the QTLs significant over at least two years, hereafter called stable QTLs: seven for berry weight, four for seed number, six for seed weight, three for seed dry matter.

\section{Berry weight}

Our results on the consensus map confirmed the existence of a major QTL for berry weight already found in seedless populations by several authors on LG 18 near the SSR marker VMC7F2; it was present only in the seedless population MTP3140, explaining 45-61\% of total phenotypic variance $\left(\sigma_{P}^{2}\right)$ depending on the year. We also found this QTL for the part of berry weight variation unexplained by seed number (RESN, 24-45\%), but not for the part unexplained by total seed weight (RESFW).

In addition to this major QTL, we discovered new, stable QTLs for berry weight on LG 8 (7-16\% of $\left.\sigma_{P}^{2}\right)$, LG 11 (10-11\%) and LG 17 (7-31\%), the latter being present in two populations. A second QTL was found on LG 18, near the VVIN83 marker ( $9 \%$ of $\sigma_{P}^{2}$ ).

The QTLs for the residual berry weight unexplained by seed number (RESN) were very similar to those for mean berry weight (MBW), whereas important differences were revealed for the residual berry weight unexplained by total seed weight (RESFW). In particular, an additional stable QTL was found for RESFW (8-18\%) on LG 13, where none was detected for MBW.

Stable berry weight QTLs showed substantially different patterns between consensus and parental maps. Noticeably, the QTL on LG 13, unstable on the consensus map, was stable for the Syrah parent.

Table 2 Broad sense heritability of seven seed and berryrelated traits in three grapevine mapping populations

\begin{tabular}{cccccccc}
\hline & MBW & MSN & TSFW & MSFW & \%SDM & RESN & RESFW \\
\hline MTP3140 & 0.83 & 0.73 & 0.95 & 0.95 & 0.94 & 0.93 & 0.89 \\
MTP3234 & 0.73 & 0.71 & 0.76 & 0.75 & 0.57 & 0.84 & 0.80 \\
SXG & 0.79 & 0.74 & 0.61 & 0.69 & - & 0.91 & 0.91
\end{tabular}

MBW: mean berry weight; MSN: mean seed number; TSFW: total seed fresh weight; MSFW: mean seed fresh weight; \%SDM: seed dry matter percentage; RESN: residual berry weight unexplained by seed number; RESFW: residual berry weight unexplained by total seed fresh weight. 
Table 3 Main QTLs for seven seed and berry-related traits in three grapevine mapping populations (consensus maps)

\begin{tabular}{|c|c|c|c|c|c|c|c|c|}
\hline Trait $^{1}$ & LG & Population & Years & $\mathrm{Cl}$ extremes & Max LOD peak & Max \% variance & Largest allelic effects ${ }^{2}$ & $\begin{array}{l}\text { QTL already published } \\
\text { (References) }\end{array}$ \\
\hline MBW & 8 & $S \times G$ & 05,06 & $21.2-56.3$ & 8.5 & 16 & $A f, D$ & No \\
\hline MBW & 11 & MTP3140 & 95,98 & $0-25.7$ & 6.6 & 11 & $A f, A m$ & No \\
\hline MBW & 17 & MTP3140 & $95,98,99$ & $18-28.3$ & 10.3 & 13 & $\mathrm{Am}, \mathrm{D}$ & No \\
\hline MBW & 17 & $S \times G$ & $05,06,07$ & $9.3-20.4$ & 17.1 & 31 & $\mathrm{Am}$ & No \\
\hline MBW & 18 & MTP3140 & $94,95,96,98,99$ & 87.6-93.3 & 38.6 & 61 & $A f, A m$ & Yes [25,27-29] \\
\hline MBW & 18 & SXG & 06,07 & $32.6-43.3$ & 6.6 & 9 & $\mathrm{Am}, \mathrm{D}$ & No \\
\hline RESFW & 8 & SxG & $05,06,07$ & $9.2-56.3$ & 10.7 & 17 & $\mathrm{Af}, \mathrm{Am}$ & No \\
\hline RESFW & 13 & $S \times G$ & $05,06,07$ & $0-32.2$ & 9.7 & 18 & $\mathrm{Af}, \mathrm{Am}$ & No (but MBW [43]) \\
\hline RESFW & 17 & $S \times G$ & $05,06,07$ & $9.3-20.4$ & 16.7 & 24 & $A f, A m$ & No \\
\hline RESFW & 18 & $S \times G$ & 06,07 & $34-43.3$ & 9.5 & 12 & $\mathrm{Am}$ & No \\
\hline RESN & 8 & $S \times G$ & 05,06 & $22.7-56.3$ & 9.5 & 15 & Af & No \\
\hline RESN & 11 & MTP3140 & $94,95,98$ & $0-33.7$ & 7.5 & 18 & $\mathrm{Af}, \mathrm{Am}$ & No \\
\hline RESN & 17 & MTP3140 & $94,95,98$ & $18-30.3$ & 8.8 & 13 & $\mathrm{Am}$ & No \\
\hline RESN & 17 & $S \times G$ & $05,06,07$ & $9.3-22.4$ & 17.8 & 29 & $\mathrm{Am}$ & No \\
\hline RESN & 18 & MTP3140 & $94,95,96,98,99$ & $84-93.3$ & 21.1 & 45 & $A f, A m, D$ & No \\
\hline RESN & 18 & $S \times G$ & 06,07 & $32.6-43.3$ & 7.2 & 10 & $\mathrm{Am}$ & No \\
\hline MSN & 2 & $S \times G$ & $05,06,07$ & $2-23.3$ & 24.8 & 48 & $A f, A m, D$ & Yes [29] \\
\hline MSN & 4 & SxG & $05,06,07$ & $47.7-56$ & 15.8 & 29 & Af & No \\
\hline MSN & 18 & MTP3140 & $94,98,99$ & $89.6-98.3$ & 24.4 & 59 & $A f, A m, D$ & Yes $[25,28]$ \\
\hline TSFW & 4 & $S \times G$ & 06,07 & $51.7-56$ & 14.2 & 29 & Af & No \\
\hline TSFW & 18 & MTP3140 & $96,98,99$ & $89.6-93.3$ & 45.6 & 82 & $\mathrm{Af}, \mathrm{Am}$ & Yes $[25,27,28]$ \\
\hline MSFW & 1 & $S \times G$ & 06,07 & $14-33.1$ & 8.0 & 15 & $A f, A m$ & No (but TSFW [27]) \\
\hline MSFW & 2 & $S \times G$ & $05,06,07$ & $0-33.3$ & 21.4 & 45 & $A m, D$ & No \\
\hline MSFW & 12 & MTP3140 & 95,99 & $0-23$ & 6.7 & 3 & $A m, D$ & No \\
\hline MSFW & 18 & MTP3140 & $94,95,98,99$ & $87.6-98.3$ & 61.4 & 87 & $A f, A m, D$ & Yes $[25,29]$ \\
\hline$\% S D M$ & 14 & MTP3234 & 03,04 & $24-41$ & 16.1 & 51 & $A m, D$ & No \\
\hline$\% S D M$ & 18 & MTP3140 & 98,99 & $87.6-93.3$ & 47.9 & 84 & Af,Am & Yes $[25,29]$ \\
\hline
\end{tabular}

QTLs derived with the MQM method. Only the QTLs found for at least two years are presented. All QTLs in this table were also significant for the BLUP of the studied trait. Confidence Interval $(\mathrm{Cl})$ extremes were the extremes of all $\mathrm{Cls}$ of both year-specific and BLUP QTLs. The genome-wide first type error rate was $a=0.05$.

${ }^{1}$ MBW: mean berry weight; MSN: mean seed number; TSFW: total seed fresh weight; MSFW: mean seed fresh weight; \%SDM: seed dry matter percentage; RESN: residual berry weight unexplained by seed number; RESFW: residual berry weight unexplained by total seed fresh weight.

${ }^{2}$ Major allelic effects: Af female, Am male, D dominance.

The stable parental QTLs for MBW were found also for RESN and/or RESFW, but four additional stable parental QTLs were found for residual values, on LGs 1, 11, 17 and 18. Among them, the QTL for RESN on LG 1 (6-9\%) revealed a new locus involved in berry weight variation that was found neither in consensus maps nor with MBW or RESFW.

No stable QTL for raw or residual berry weight data was found on consensus or parental maps in population MTP3346.

\section{Seed traits}

For mean seed number per berry (MSN), mean seed fresh weight per berry (MSFW), total seed fresh weight per berry (TSFW) and seed dry matter percentage (\%SDM), we confirmed the already described major QTL on LG 18 near VMC7F2, that explained up to 59\%, 87\%, 82\% and $35 \%$ of the total variance, respectively.

For MSN, we confirmed a QTL previously found on LG $2(12-48 \%)$ and discovered two additional stable QTLs, on LG 4 (14-29\%) and LG 14 (17-23\%, in MTP3346). For MSFW, three new stable QTLs were discovered on consensus maps on LG 1 (9-15\%), LG 2 (13-45\%) and LG 12 (3\%). For TSFW, two new QTLs were detected, on LG 4 (22-29\%, both consensus and parental) and LG 13 (9-11\%, parental only). For \%SDM, two other QTLs were uncovered, on LG 5 (17-31\%, in MTP3346) and LG 14 (35-46\%). 
Table 4 Main QTLs for seven seed and berry-related traits in three grapevine mapping populations (parental maps)

\begin{tabular}{|c|c|c|c|c|c|c|c|c|}
\hline Trait $^{1}$ & LG & Population & Map & Years & $\mathrm{Cl}$ extremes & Max LOD peak & Max \%var & $\begin{array}{l}\text { QTL already published } \\
\text { (References) }\end{array}$ \\
\hline$\overline{M B W}$ & 8 & SG & $\mathrm{S}$ & $05,06,07^{2}$ & $0.0-37.4$ & 9.4 & 20 & No \\
\hline MBW & 13 & SG & S & 05,06 & $0.0-13.3$ & 5.9 & 17 & Yes [43] \\
\hline MBW & 17 & SG & G & $05,06,07$ & $9.9-18.3$ & 15.0 & 25 & No \\
\hline MBW & 18 & MTP3140 & $\mathrm{F}$ & $94,95,96,98,99$ & $51.0-58.2$ & 16.3 & 37 & Yes [25,27-29] \\
\hline MBW & 18 & MTP3140 & M & $94,95,96,98,99$ & $101.5-115.3$ & 12.7 & 32 & Yes [25,27-29] \\
\hline RESFW & 8 & SG & S & $05,06,07$ & $0.0-33.4$ & 8.4 & 20 & No \\
\hline RESFW & 13 & SG & S & 05,07 & $0.0-12.0$ & 5.5 & 14 & No (but MBW [43]) \\
\hline RESFW & 17 & SG & G & $05,06,07$ & $9.9-18.3$ & 15.3 & 24 & No \\
\hline RESFW & 17 & MTP3140 & M & $94,95,98,99$ & $17.5-31.2$ & 5.7 & 15 & No \\
\hline RESFW & 18 & SG & G & $05,06,07$ & $24.2-49.7$ & 5.7 & 10 & No \\
\hline RESN & 1 & SG & G & 05,06 & $12.1-41.9$ & 4.9 & 9 & No \\
\hline RESN & 8 & SG & S & $05,06,07$ & $0.0-39.4$ & 8.7 & 20 & No \\
\hline RESN & 11 & MTP3140 & $\mathrm{F}$ & 94,96 & $10.1-22.9$ & 6.0 & 20 & No \\
\hline RESN & 17 & SG & G & $05,06,07$ & $8.0-18.3$ & 14.3 & 23 & No \\
\hline RESN & 17 & MTP3140 & M & $94,95,98$ & $17.5-31.2$ & 6.2 & 16 & No \\
\hline RESN & 18 & MTP3140 & $\mathrm{F}$ & $95,98,99$ & $51.0-76.9$ & 8.2 & 22 & No \\
\hline RESN & 18 & MTP3140 & M & $94,95,98,99$ & 89.9-115.3 & 8.7 & 22 & No \\
\hline MSN & 2 & SG & G & 05,07 & $4.0-25.9$ & 7.8 & 20 & Yes [29] \\
\hline MSN & 4 & SG & S & $05,06,07$ & $40.3-48.3$ & 13.6 & 26 & No \\
\hline MSN & 18 & MTP3140 & $\mathrm{F}$ & $94,95,96,98$ & $40.8-72.9$ & 11.5 & 29 & Yes $[25,28]$ \\
\hline MSN & 18 & MTP3140 & M & $94,96,98$ & $113.5-115.3$ & 6.5 & 17 & Yes $[25,28]$ \\
\hline TSFW & 4 & SG & S & $05,06,07$ & $42.3-48.3$ & 16.5 & 32 & No \\
\hline TSFW & 13 & SG & G & $05,07^{2}$ & $13.6-46.4$ & 4.7 & 11 & No (but MSFW [29]) \\
\hline TSFW & 18 & MTP3140 & $\mathrm{F}$ & $94,95,96,98,99$ & $51.0-58.2$ & 20.3 & 44 & Yes $[25,27,28]$ \\
\hline TSFW & 18 & MTP3140 & M & $94,95,96,98$ & $113.5-115.3$ & 16.5 & 40 & Yes $[25,27,28]$ \\
\hline MSFW & 2 & SG & G & 05,07 & $0.0-17.0$ & 9.1 & 23 & No \\
\hline MSFW & 18 & MTP3140 & $\mathrm{F}$ & $95,98,99$ & $51.0-56.6$ & 19.3 & 43 & Yes $[25,29]$ \\
\hline MSFW & 18 & MTP3140 & M & $94,95,96,98^{2}$ & $101.5-115.3$ & 12.4 & 35 & Yes $[25,29]$ \\
\hline$\% S D M$ & 18 & MTP3140 & $\mathrm{F}$ & $94,95,96,98,99$ & $46.7-58.2$ & 17.8 & 41 & Yes $[25,29]$ \\
\hline$\% S D M$ & 18 & MTP3140 & M & $94,95,96,99^{2}$ & $101.5-115.3$ & 13.6 & 37 & Yes $[25,29]$ \\
\hline
\end{tabular}

QTLs derived with the CIM method. Only the QTLs found for at least two years are presented. All QTLs in this table were also significant for the BLUP of the studied trait, except those indicated by ${ }^{2}$. Confidence Interval (CI) extremes were the extremes of all Cls of both year-specific and BLUP QTLs. The genome-wide first type error rate was $a=0.05$.

M: male; F: female; S: Syrah; G: Grenache.

${ }^{1}$ MBW: mean berry weight; MSN: mean seed number; TSFW: total seed fresh weight; MSFW: mean seed fresh weight; \%SDM: seed dry matter percentage; RESN: residual berry weight unexplained by seed number; RESFW: residual berry weight unexplained by total seed fresh weight.

${ }^{2} \mathrm{QTL}$ not significant for the BLUP of the studied trait.

\section{Candidate genes}

Despite having reduced explored intervals to a distance of $+/-3 \mathrm{cM}$ around the LOD peak, the number of positional candidate genes found remained very large, varying from 77 to 167 across QTL regions (Additional file 14: Table S10). However, only 11 corresponded to functional candidate genes involved in cell wall modifications, water transport, transcription regulation, organ identity, ethylene and auxin signalling in grapevine. Three additional ones belonged to the gene families with an established role in fruit weight in tomato (Table 5 and Additional file 15: Table S11). Indeed, the recent cloning of the fruit weight QTL fw3.2 in Solanum lycopersicum (cultivated tomato) [58] led to the identification of the LOC101258933 gene, encoding a cytochrome $\mathrm{P} 450$ 78A-like protein. In the grapevine genome, eight genes are annotated as P450 78A-like proteins (NCBI). Noticeably, the putative ortholog of LOC101258933, displaying $75 \%$ identity on $99 \%$ of the sequence length (LOC100253660, LG17:5600143..5602650), 


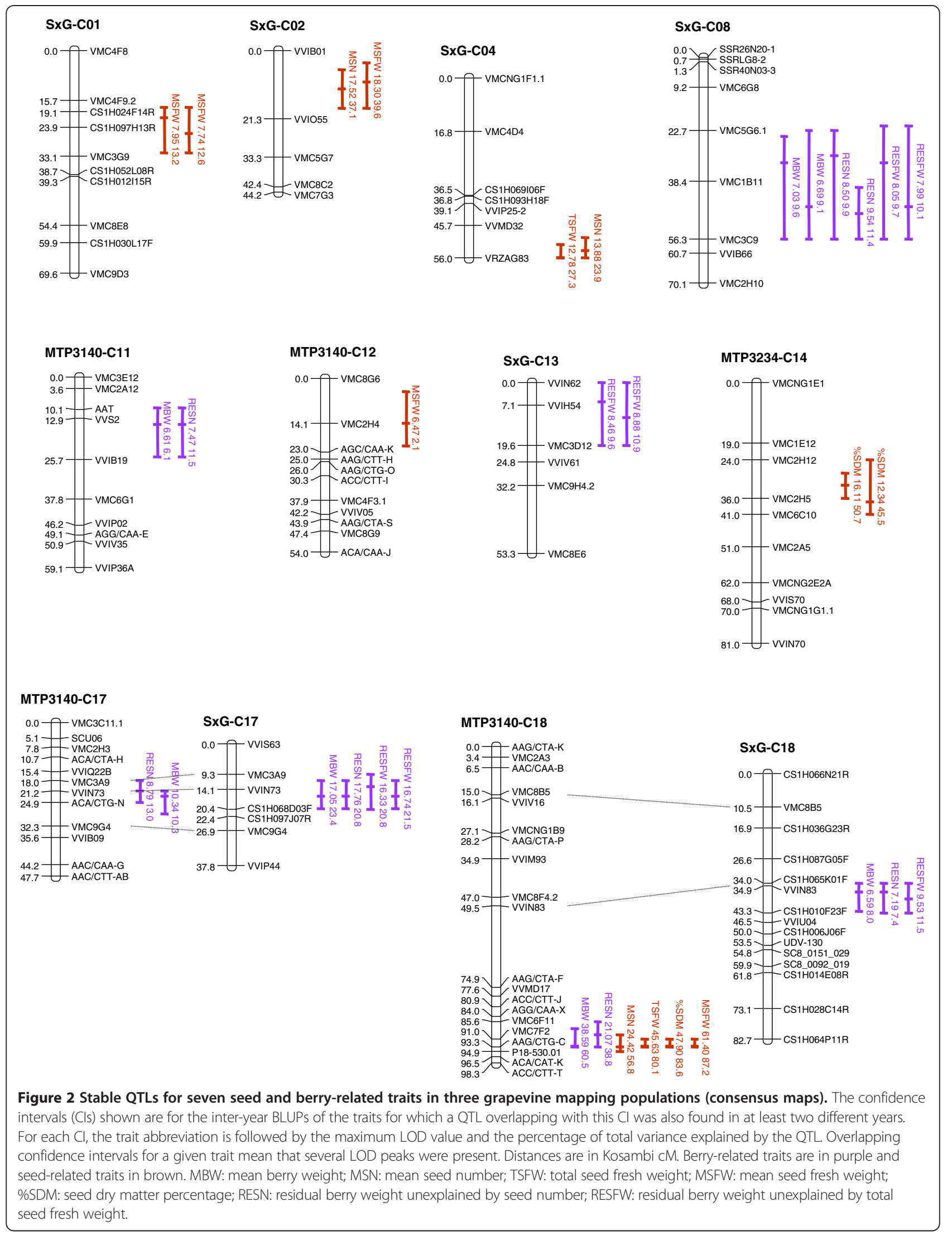


Table 5 Published functional candidate genes potentially involved in grapevine seed and/or berry development colocalized with QTLs detected

\begin{tabular}{|c|c|c|c|c|c|c|}
\hline $\begin{array}{l}\text { Grapevine } 12 X \\
\text { gene ID }\end{array}$ & Gene name & LG & position in $12 X$ & $\begin{array}{l}\text { putative homologous gene } \\
\text { and/or function }\end{array}$ & $\begin{array}{l}\text { In the } \mathrm{Cl} \text { of a } \\
\text { QTL of the } \\
\text { present study }{ }^{1}\end{array}$ & Reference \\
\hline GSVIVT01011687001 & - & 1 & 51230905124512 & $\begin{array}{l}Z m C N R 2 \text { involved in tissue growth } \\
\text { activity in maize }\end{array}$ & RESN, MSFW & {$[60]$} \\
\hline GSVIVT01018839001 & VVAP3.2 = TM6 & 4 & 1939543819397804 & MIKC gene expressed in flowers and berries & MSN, TSFW & [61] \\
\hline GSVIVT01025701001 & - & 8 & 1284344212845830 & $\begin{array}{l}\text { ethylene signalling protein preferentially } \\
\text { expressed in flb mutant vs WT }\end{array}$ & MBW - RESFW & {$[62]$} \\
\hline GSVIVT01025700001 & & & 1284583612854444 & & & \\
\hline \multirow[t]{2}{*}{-} & \multirow[t]{2}{*}{-} & \multirow[t]{2}{*}{8} & 13327417 & \multirow[t]{2}{*}{ cell number regulator 8-like } & \multirow{2}{*}{$\begin{array}{l}\text { MBW, RESN, } \\
\text { RESFW }\end{array}$} & \multirow[t]{2}{*}{-} \\
\hline & & & 13332631 & & & \\
\hline GSVIVT01032681001 & EXP2 & 13 & 15786451580497 & $\begin{array}{l}\text { expansin EXP8 (A. thaliana) with expression } \\
\text { linked to berry development }\end{array}$ & $\begin{array}{l}\text { MBW, RESFW } \\
\text { (in Syrah) }\end{array}$ & [63] \\
\hline GSVIVT01016525001 & EXP3 & 13 & 31202773122170 & $\begin{array}{l}\text { expansin EXP4 (A. thaliana) with expression } \\
\text { linked to berry development }\end{array}$ & $\begin{array}{l}\text { MBW, RESFW } \\
\text { (in Syrah) }\end{array}$ & [63] \\
\hline GSVIVT01016276001 & $\mathrm{AQ} 2=P I P 2 ; 1$ & 13 & 56020255605019 & $\begin{array}{l}\text { Plasma membrane intrinsic protein 2B (PIP2B); } \\
\text { aquaporin PIP2.2 (A. thaliana) with expression } \\
\text { linked to berry development }\end{array}$ & RESFW & [63] \\
\hline GSVIVT01008122001 & - & 17 & 56001435602650 & $\begin{array}{l}\text { Cytochrome P450 78A-like protein in } \\
\text { Solanum lycopersicum (tomato), harboring } \\
\text { the causal SNP of a berry weight QTL }\end{array}$ & $\begin{array}{l}\text { MBW-RESN- } \\
\text { RESFW }\end{array}$ & {$[58]$} \\
\hline GSVIVT01008046001 & - & 17 & 63161686320317 & $\begin{array}{l}\text { WRKY transcription factor } 72 \text {-like, } \\
\text { regulation of skin and flesh ripening }\end{array}$ & $\begin{array}{l}\text { MBW, RESN, } \\
\text { RESFW }\end{array}$ & [64] \\
\hline GSVIVT01008034001 & - & 17 & 64555256456835 & $\begin{array}{l}\text { transcription factor bHLH135-like, } \\
\text { regulation of pre-véraison processes } \\
\text { in the pericarp }\end{array}$ & $\begin{array}{l}\text { MBW, RESN, } \\
\text { RESFW }\end{array}$ & [64] \\
\hline GSVIVT01007987001 & EXPA & 17 & 68883736890086 & $\begin{array}{l}\text { Alpha-expansin with expression linked } \\
\text { to berry development }\end{array}$ & $\begin{array}{l}\text { MBW }{ }^{2}, \text { RESN }^{2}, \\
\text { RESFW' }\end{array}$ & {$[65]$} \\
\hline GSVIVT01009791001 & VVBG1 & 18 & 1128657811293205 & $\begin{array}{l}\text { beta-galactosidase (cell-wall modifying } \\
\text { enzyme expressed during berry } \\
\text { development) }\end{array}$ & $\begin{array}{l}\text { MBW, RESN, } \\
\text { RESFW }\end{array}$ & {$[66]$} \\
\hline \multirow[t]{3}{*}{ GSVIVG01009815001 } & \multirow[t]{3}{*}{ VVAP3 } & \multirow[t]{3}{*}{18} & \multirow[t]{3}{*}{1150651411512366} & $\begin{array}{l}\text { Apetala } 3 \text { (Arabidopsis), MADS box flower } \\
\text { development }\end{array}$ & \multirow[t]{3}{*}{$\begin{array}{l}\text { MBW, RESN, } \\
\text { RESFW }\end{array}$} & [67] \\
\hline & & & & expressed highest in young fruit & & {$[68]$} \\
\hline & & & & $\begin{array}{l}\text { expressed almost exclusively in } \\
\text { inflorescences }\end{array}$ & & [61] \\
\hline GSVIVT01009865001 & - & 18 & 1192049811929437 & $\begin{array}{l}\text { auxin response factor } 5 \text {-like, transcript } \\
\text { variant } 1 \text {, includes an EST of hypothetical } \\
\text { transcription factor preferentially expressed } \\
\text { in WT vs flb mutant }\end{array}$ & RESFW & {$[62]$} \\
\hline GSVIVG01025945001 & $\begin{array}{l}\text { VVAGL11 = VVAG3 }= \\
\text { MADS5 }\end{array}$ & 18 & 2688867726896544 & $\begin{array}{l}\text { Agamous like } 11 \text { (Arabidopsis), MADS box } \\
\text { ovule identity (MIKC gene expressed in } \\
\text { flowers and berries) }\end{array}$ & $\begin{array}{l}\text { MBW, RESN, } \\
\text { MSN, TSFW, } \\
\text { MSFW,\%SDM }\end{array}$ & {$[30,67]$} \\
\hline
\end{tabular}

Candidate genes colocalized with QTLs detected in the present study. Gene numbers were obtained from the automatic annotation provided with the Genoscope 12X whole genome sequence release of PN40024 (http://www.genoscope.cns.fr/externe/GenomeBrowser/Vitis/ [1]).

${ }^{1}$ MBW: mean berry weight; MSN: mean seed number; TSFW: total seed fresh weight; MSFW: mean seed fresh weight; \%SDM: seed dry matter percentage; RESN: residual berry weight unexplained by seed number; RESFW: residual berry weight unexplained by total seed fresh weight.

${ }^{2}$ In QTL confidence interval but not within $3 \mathrm{cM}$ of LOD peak position.

colocalized with the major berry weight QTL, while the second matching gene did not colocalize with a QTL (LOC100249034, LG1:381267..382921, 71\% identity on 99\% of the sequence).

The cloning of another fruit weight QTL in tomato, fw2.2 [69], led to the identification of the LOC101245309 gene, encoding a cell number regulator. The three best matches on Vitis refseq database were on LG3, where no QTL was found. However, three other genes were annotated as predicted cell number regulator-like in grapevine genome, among which LOC100246592 colocalized with a berry size QTL, on LG8 (Additional file 16: Table S12). In 
addition, the best match of the maize $Z m C N R 2$ gene from the same family, potentially involved in tissue growth [60], was obtained for LOC100250939, encoding a plant cadmium resistance 2-like protein, which colocalized with the parental-only berry growth QTL on LG1.

Many other positional candidate genes with putative functions similar to the functions of these 14 genes were found under berry weight QTLs, but no published study provided evidence of any function related to grapevine berry development (Additional file 16: Table S12).

\section{Discussion}

\section{Genetic architecture of berry weight and seed content Detection of new QTLs}

This study reports five new QTLs for berry weight in grapevine, located on LGs 1, 8, 11, 17 and 18, and stable over at least two years. The only stable QTLs previously known for this trait were a major one linked to seedlessness on LG 18 [25,27-29] and two QTLs on LGs 5 and 13 found in a complex hybrid cross including non-vinifera background [43]. The second QTL for berry weight found on LG 18, near the VVIN83 marker, was stable over two years in our study, whereas it was found unstable by Cabezas et al. [27]. All these novel QTLs were stable over time but not over populations, except the QTL with the largest effect on LG 17, found in two populations with distant genetic backgrounds. This is consistent with the highly composite nature of berry weight, affected by numerous factors (cell multiplication, cell wall modifications, photosynthesis, sucrose and water transport, growth regulators, etc.), and therefore expected to be under polygenic control, with different causal polymorphisms segregating in different populations.

The present work is the first report of a stable QTL for seed traits in a seeded wine grape background, on LG 4. New QTLs for seed traits in seedless and/or table backgrounds were also found, on LGs 5, 12 and 14.

A number of methodological choices have allowed us to make improvements over previous studies and therefore to find additional QTLs and to provide new insights for already known ones. First, the simultaneous study of four populations using shared methods for phenotyping, genotyping and statistical analyses facilitated result comparisons. In previous studies (Additional file 1: Table S1), comparisons were made difficult by differences in thresholds (genome-or chromosome-wide), maps (parental or consensus) and studied traits, even if most studies used a common phenotyping protocol (European project MASTER, ended in 2005). Several QTLs could not have been found using parental maps only as in [25] (Tables 3 and 4). Most of these showed dominance allelic effects on the consensus map. However, the study of parental maps proved to remain necessary by revealing QTLs otherwise unstable on the consensus map. This could result from a higher power of additive QTL detection in parental maps, where the sample size of each genotypic class is twice as large as in the consensus map. Second, composite interval mapping allowed to discover additional QTLs, compared to interval mapping only as in [28], such as the berry weight QTL on LG 17, detected after adding the major QTL on LG18 as a cofactor in population MTP3140. Third, by analysing mean seed fresh weight in addition to total seed fresh weight, three additional QTLs could be discovered. Moreover, the absence of QTL for mean seed weight on LG 4 suggests that the only QTL for total seed weight on this LG was most probably due to the QTL for seed number. This clearly emphasizes the need to study elementary components of complex traits in QTL detection. Fourth, searching for QTLs using BLUPs of the traits of interest in addition to QTLs in individual years provides useful summarized information. Despite its demonstrated interest in other plant species [70], the use of BLUPs in QTL studies on grapevine is very recent $[49,71,72]$ and was applied here to berry weight or seed traits for the first time. Last, we provide here the first attempt to search for QTLs using the residual part of berry weight unexplained by seed trait variation. This analysis successfully revealed two stable QTLs for residual berry weight on LGs 1 and 13, that were not detected with berry weight raw data. The QTL found on LG 1 had never been reported before.

\section{Confirmed or invalidated QTLS}

We found only in the seedless population MTP3140 the major QTL on LG 18 for berry weight and seed traits previously found in several studies [25,27-29]. In the second seedless population MTP3346, the parents were homozygous around this QTL, therefore no segregation occurred. In this region, no stable QTL was found for the residual part of berry weight variation not explained by total seed weight, whereas a stable QTL was found for the part unexplained by seed number alone. This difference could be due to insufficient power to detect QTLs with a low residual variation since the genetic correlation between berry weight and total seed weight was very high. Alternatively, it could suggest that this major berry weight QTL is a pleiotropic QTL for both mean seed fresh weight and berry weight, with direct or indirect effects via growth regulator production by seeds, as argued by Meijia et al. [30].

The stable QTLs found here were consistent with several previously reported QTLs for related traits, and their comparison provided more insight into their determinism. On LG 1, Cabezas et al. [27] found a stable QTL for total seed fresh weight that overlapped the QTL for mean seed fresh weight in our study, suggesting that the underlying gene might control total seed weight through individual seed weight (not studied by these authors) rather than through seed number. On LG 13, 
our QTL for berry weight colocalized with the stable one found by Fisher et al. [43] in a complex interspecific background. In the same region, we also found a QTL for the residual berry weight unexplained by total seed fresh weight, which suggests that this berry weight QTL was not only due to variation in seed content. On LG 13, we detected a QTL for total seed fresh weight where Costantini et al. [29] found a QTL for mean seed fresh weight, which is consistent since these authors did not study total seed fresh weight, and which suggests that our QTL for total seed fresh weight is explained by mean seed weight rather than by seed number.

On LG 2, the QTLs detected for seed number and mean seed fresh weight colocalized with the QTL for seed number in Costantini et al. [29]. However, in a further analysis to include flower sex morphology (females $v s$ hermaphrodites) as a covariate into the QTL detection model using the R/qtl package [73], our large seed QTLs on LG 2 were not detected (data not shown). This suggests these QTLs were not true QTLs for seed traits but corresponded to the major locus for sex mapped on this LG by several authors [74-77]. The SxG population segregated for sex since both parents were heterozygous HF (hermaphrodite-female). The effect of sex on both seed number and mean seed fresh weight was highly significant each year $(P<0.001)$. The QTL for seed number reported by Costantini et al. [29] on LG 2 is most probably also explained by the sex locus which segregated in their mapping population (L. Costantini, pers. comm.). To our knowledge, there is no published evidence of an effect of sex on seed number or weight in grapevine, even though seed number in female plants is expected to be lower than in hermaphrodite ones as suggested by the obligatory outcrossing and higher rate of parthenocarpic berries set [78]. Interestingly, though female varieties usually have rather large berries [3], we found no QTL for berry weight on LG 2 and the effect of sex on berry weight was never significant in the SxG population for this trait.

Only two stable QTLs for berry weight previously published, on LGs 5 and 10, were not found here. This highlights the large genetic variability explored in our study by involving eight different parents. A total of at least six stable minor QTLs for seed traits in seedless background were found in this study and/or in previous studies (Additional file 1: Table S1). Therefore, the genetic model for seedlessness proposed by Bouquet and Danglot [46], with a major gene on LG 18 regulating three minor genes, needs to be complexified.

Only one previously reported unstable QTL [27] was found stable in one of our populations (on LG 18 near VVIN83). All other published unstable QTLs (Additional file 1: Table S1) were not confirmed in our study, emphasizing the low potential interest of such QTLs interacting with environment. Therefore, we chose not to discuss unstable QTLs, even those concerning regions not identified previously.

\section{Heritability}

The relatively high values of broad sense heritabilities found for berry and seed weights in the four populations (0.51-0.93 and 0.61-0.95, respectively) were consistent with previously published results [39-42]. However, heritability for seed number was much higher in our study than in Daulta et al. [39] ( $\geq 0.59$ vs 0.34). The allelic effects of all QTLs found here had at least one additive component, and about two thirds of the QTLs did not show any large dominance effect (Table 3 and Additional file 8: Table S6). This is consistent with the high values of narrow sense heritabilities (around 0.60) reported for both berry weight and seedlessness $[31,32,38]$.

\section{Relationship between berry weight and seed traits}

Most stable QTLs found here for berry weight and seed traits did not colocalize. In particular, the seed QTL on LG 5 did not colocalize with the berry weight QTL found by Fisher et al. [43]. Moreover, it is worth noting that no colocalization was found with the two minor QTLs for seed number. Nevertheless, colocalization was observed in three regions. The first case concerned the major QTL on LG 18 in the MTP3140 seedless population. This is consistent with the high genetic correlation observed in this population and the weaker correlation obtained for the other populations with no QTL found in this part of LG 18. This result is also consistent with the strong correlations previously reported in segregating populations or collections involving seedless genotypes [24-32], mainly derived from cv. Sultanina, and the absence of correlation reported in collections composed mainly of seeded cultivars $[4,33]$. In the seeded population SxG, the extent of variation in seed traits was smaller compared to MTP3140. However, this alone could not explain the lower berry-seed correlation, since variation was large in MTP3234. The second case of berry-seed QTL colocalization was on LG 1. But the consensus QTL for mean seed weight had both cvs. Syrah and Grenache additive effects, whereas the QTL for residual berry weight was stable only in Grenache. This indicates that colocalization could not result only from a pleiotropic effect. The third colocalization was found on LG 13 with a QTL for total seed weight in cv. Grenache. In that case also, pleiotropy alone could not explain colocalization because additive effects had different parental origins.

From an evolutionary point of view, the wild $V$. vinifera subsp. sylvestris has much smaller berries than the cultivated $V$. vinifera subsp. sativa but seeds of similar size $[79,80]$. Therefore, the anthropic selection for larger 
berry sizes was probably based on QTLs for berry size unrelated to seed traits, such as the four ones detected in this study. Quite interestingly, the QTL on LG 17 colocalized with the $5-\mathrm{Mb}$ candidate domestication locus on chromosome 17 found by Myles et al. [81]. If selection occurred during primary domestication, a drastic reduction in genetic diversity would be expected at this locus in cultivated grapevine. However, small berry size alleles may have been re-introduced afterwards during secondary phases of domestication $[82,83]$, or mutations may have arisen after domestication, which could explain the detection of this QTL in two different populations. Alternatively, it cannot be excluded that these two populations harbor rare alleles.

In the future, these limited colocalizations between QTLs for berry size and seed traits offer the possibility to reduce seed number and size without reducing berry size using Marker Assisted Selection (MAS) to accelerate table grape breeding. However, the existence of a threshold in seed content, under which the potential berry size would be limited, could prevent the complete dissociation of these traits and therefore the breeding of cultivars with very large berries and seeds imperceptible enough for the consumers.

The role in grape berry development of growth regulators produced by seeds was questioned by Mullins et al. [6]. It is generally accepted that embryos control cell division in the surrounding fruit tissues [84]. In grape, Mullins et al. [6] argued that growth regulators produced by seeds might not play a major role in berry development pattern in grapevine, since the relationship between mean growth patterns of seeds and berries is highly variable among seeded cultivars. Sugar accumulation or cell wall extensibility could better explain grape berry growth pattern. However, Ojeda et al. [7] suggested that seed growth affects more profoundly berry cell mitosis than cell enlargement. The distinct QTLs found for seed and berry traits in our study strengthen Mullins et al.'s hypothesis [6], since berry QTL intervals harbored several genes involved in transport or cell wall modifications (see below). Alternatively, QTLs for berry size that did not colocalize with QTLs for seed content might be QTLs for some other internal factors related to berry size at maturity, such as growth regulators not produced by seeds or sink/source ratio [9]. Measurements of these traits on the same populations would be required to assess the extent of their genetic relationship with berry weight at maturity.

\section{Candidate genes}

Several hundred positional candidate genes were found under QTLs (Additional file 14: Table S10). However, only a few genes deserve particular attention (Table 5), because they could be either potential orthologs of genes underlying tomato fruit weight QTLs, or involved in berry weight or seed content based on functional evidence in grapevine.

A cytochrome P450 78A gene partly controlling fruit weight in tomato was suspected to be involved in the domestication of this species [58]. The colocalization between the putative ortholog of this gene and the large berry size QTL on LG17 suggests a rather strict conservation of the control of fruit size across such physiologically distant fruit species. Selection of large-fruit alleles at this locus during the domestication process is also possible in grapevine since it colocalizes with a putative domestication locus [81]. Colocalization was also observed between the berry weight QTL on LG8 and a gene from the CNR family, which regulates cell number and was involved in fruit size changes during the domestication of tomato [69]. De Franceschi et al. [57] also suggested the implication of a gene from this family in the domestication of sweet cherry.

Other functions, involved in cell expansion during berry development are likely to be responsible for grapevine berry weight variation, in the first place concerning the increase in cell wall area. Schlosser et al. [63] showed that a cohort of candidate cell wall-modifying enzymes (expansin, glycosyl hydrolase, pectinesterase, pectate lyase, cellulase, XET) were highly expressed during berry growth. The two expansin isogenes that these authors studied, EXP2 and EXP3, fell under the berry weight QTL on LG 13. An alpha-expansin with expression linked to berry development has also been found in the confidence interval of the berry weight QTL on LG 17 [65]. On LG 18, a beta-galactosidase gene expressed during berry development colocalized with the minor berry weight QTL [66]. This enzyme showed the most dramatic change in activity amongst cell wall enzymes during berry ripening, probably linked to the increase in pectin solubility [85].

Water import, mediated by MIP and PIP genes, is also essential for cell expansion. On LG 13, the putative aquaporine VvPIP2;1 isogene, up-regulated at the beginning of the second growth phase [63], was located under the QTL for residual berry weight. An EST-derived probe of PIP2-1 type was found over-expressed after GA-treatment around véraison [86]. A putative paralog with similar function, Refseq VvPIP2;3, represents the best hit of EF364437.1 in the $12 \mathrm{X}$ genome and is located under the QTL for berry weight on LG 8. VvPIP2;1 and VvPIP2;3 actually triggered large water channel activities upon expression in Xenopus oocytes [87]. These genes were highly expressed in expanding green berries, according to EST counts at NCBI, as confirmed by RNAseq [88].

Many other genes could also be considered as potential candidate genes for berry weight QTLs, even when no precise function has been identified for them directly 
in grapevine yet (Additional file 16: Table S12). These genes are those expected to control processes involved in fruit expansion, such as auxin and gibberellin signalling [16], source (leaf area) to sink (fruit weight) ratio [89], sugar and water balance [9], sugar biosynthesis and transport [65,90], scion/rootstock interaction, cell division or elongation, transcription control [64], signal transduction [90], or organ identity. In particular, the gene coding for an ERD6-like putative sugar transporter (GSVIVT01009719001), could be relevant under the berry weight QTL on LG 18 near the VVIN83 SSR. Lastly, it should be noted that $V v G A I 1$, a negative regulator of gibberellin response, although present in the confidence interval of the QTL on LG 1, was not expressed in berries and berry weight was not impaired in the vvgai1 loss-of-function mutant [91].

\section{Conclusions}

In four grapevine seeded and seedless mapping populations, we identified nine new QTLs for berry weight and seed content, which were stable over time. With this single study, we thus increased more than twofold the number of stable QTLs for these traits known to date. Most berry and seed QTLs did not colocalize, providing new insight into the complex correlation between berry size and seed content. Such uncolocalized QTLs could be used in marker-assisted breeding. A few candidate genes under these QTLs were functionally relevant and could readily be further tested by association genetics. However, more than one out of four genes under the QTLs had unknown functions (data not shown). Since over 17,000 genes are known to be expressed during berry development, of which nearly one third are stage-specific [88], expression patterns alone will not be very helpful for further candidate gene screening. For complex traits such as berry weight and seed content at maturity, fine mapping of QTLs and additional functional data are required to discover their causal polymorphisms.

\section{Additional files}

Additional file 1: Table S1. Published QTLS for berry weight and seed traits in Vitis.

Additional file 2: Table S2. Main features of the framework genetic maps used for QTL detection in four grapevine mapping populations.

Additional file 3: Figure S1. Raw phenotypic data distributions for five seed and berry-related traits in four grapevine mapping populations.

Additional file 4: Table S3. Transformations applied to raw data and model selected to estimate BLUPs of genetic values and broad-sense heritability, for seven seed and berry-related traits in four grapevine mapping populations.

Additional file 5: Table S4. Phenotypic correlations between years for seven seed and berry-related traits in four grapevine mapping populations (Spearman correlation coefficient).
Additional file 6: Figure S2. Genetic correlations (among BLUPS of the genetic value) between seven seed and berry-related traits within years in the grapevine mapping population MTP3346.

Additional file 7: Table S5. Broad sense heritability of seven seed and berry-related traits in the grapevine mapping population MTP3346.

Additional file 8: Table S6. Summary of main QTLs for seven seed and berry-related traits in the grapevine mapping population MTP3346 (consensus map), derived with the MQM method.

Additional file 9: Table S7. Summary of main QTLs for seven seed and berry-related traits in the grapevine mapping population MTP3346 (parental maps), derived with the CIM method.

Additional file 10: Table S8. Detailed features of all QTLs for seven seed and berry-related traits in four grapevine mapping populations (consensus maps), derived with the MQM method.

Additional file 11: Table S9. Detailed features of all QTLs for seven seed and berry-related traits in four grapevine mapping populations (parental maps), derived with the CIM method.

Additional file 12: Figure S3. Stable QTLS for seven seed and berryrelated traits in the grapevine mapping population MTP3346 (consensus map). Additional file 13: Figure S4. Stable QTLs for seven seed and berryrelated traits in four grapevine mapping populations (parental maps).

Additional file 14: Table S10. Intervals explored and number of positional candidate genes found.

Additional file 15: Table S11. Published functional candidate genes potentially involved in seed and/or berry development not colocalized with QTLs detected in the present study [92-106].

Additional file 16: Table S12. Functional candidate genes comprised within $+/-3$ cM of the LOD peaks of consensus berry size QTLs (BLUPs only).

\section{Abbreviations}

BIC: Bayesian Information Criterion; BLUP: Best Linear Unbiased Predictor; Cl: Confidence interval; CIM: Composite interval mapping; CTIFL: Centre Technique Interprofessionnel des Fruits et Légumes; IM: Interval mapping; INRA: Institut National de la Recherche Agronomique; KW: Kruskal-Wallis; LG: Linkage group; MAS: Marker assisted selection; MBW: Mean berry weight; MQM: Multiple QTL Model; MSFW: Mean seed fresh weight; MSN: Mean seed number; NCBI: National Center of Biotechnology Information; QTL: Quantitative trait locus; RESFW: Residual berry weight unexplained by total seed fresh weight; RESN: Residual berry weight unexplained by seed number; MAS: Marker assisted selection; \%SDM: Seed dry matter percentage; SSR: Simple sequence repeat; TSFW: Total seed fresh weight.

\section{Competing interests}

The authors declare that they have no competing interests.

\section{Authors' contributions}

AD conceived and designed the study, contributed to the phenotyping of the populations, managed the cultivation and phenotyping, performed statistical and bioinformatical analyses and drafted the manuscript. YB, MG and $G B$ were in charge of the cultivation of the populations in the field and greenhouse. YB, MF, MG, GB, FE, SD, PF, TP, PO, CaR, VL, RB and JPP participated in the phenotyping of the populations. ChR helped with bioinformatic analyses and discussion on candidate genes. $\mathrm{CH}$ helped searching for functional candidate genes. VL, RB, ChR, JPP and PT participated in the discussion of results and helped to draft the manuscript. PT coordinated the study and participated in phenotyping. All authors read and approved the final manuscript.

\section{Acknowledgements}

This work was supported by the European Union [grant numbers ICA4-200110065 MASTER project, 513960 FLAVO project] and the Institut National de la Recherche Agronomique. We thank the CTIFL for growing the MTP3346 population in their greenhouse, the INRA experimental station of Vassal for the controlled crosses and the first growing of ungrafted populations in the field, the INRA experimental station of Chapitre for subsequent growing in the field, Alain Bouquet for creating, managing and partly phenotyping the MTP3140 population, World Wine Vineyards for helping with overgrafting, 
all the members of the research team involved in phenotyping, Sandrine Lalet for sex phenotyping and Philippe Chatelet for English editing and useful suggestions. We thank the three anonymous reviewers and the editor for their useful comments on a first version of this manuscript.

Received: 12 July 2013 Accepted: 10 December 2013 Published: 19 December 2013

\section{References}

1. Jaillon O, Aury JM, Noel B, Policriti A, Clepet C, Casagrande A, Choisne N, Aubourg S, Vitulo N, Jubin C, Vezzi A, Legeai F, Hugueney P, Dasilva C, Horner D, Mica E, Jublot D, Poulain J, Bruyere C, Billault A, Segurens B, Gouyvenoux M, Ugarte E, Cattonaro F, Anthouard V, Vico V, Del Fabbro C, Alaux M, Di Gaspero G, Dumas V, et al: The grapevine genome sequence suggests ancestral hexaploidization in major angiosperm phyla. Nature 2007, 449:463-467.

2. Velasco R, Zharkikh A, Troggio M, Carthwright DA, Cestaro A, Pruss D, Pindo M, FitzGerald LM, Vezzulli S, Reid J, Malacarne G, lliev D, Coppola G, Wardell B, Micheletti D, Macalma T, Facci M, Mitchell JT, Perazzolli M, Eldredge G, Gatto P, Oyzerski R, Moretto M, Gutin N, Stefanini M, Chen Y, Segala C, Davenport C, Demattè L, Mraz A, et al: A high quality draft consensus sequence of the genome of a heterozygous grapevine variety. PLOS ONE 2007, 12:e1326.

3. Boursiquot JM, Dessup M, Rennes C: Distribution des principaux caractères phénologiques, agronomiques et technologiques chez Vitis vinifera L. Vitis 1995, 34:31-35.

4. Houel C, Martin-Magniette ML, Nicolas SD, Lacombe T, Le Cunff L, Franck D, Torregrosa L, Conéjéro G, Lalet S, This P, Adam-Blondon AF: Genetic variability of berry size in the grapevine (Vitis vinifera L.). Aust J Grape Wine Res 2013, 19:208-220.

5. Coombe BG: The development of fleshy fruits. Annu Rev Plant Physiol 1976, 27:507-528.

6. Mullins MG, Bouquet A, Williams LE: Developmental physiology: flowering and fruiting. In Biology of the grapevine. Edited by Mullins MG, Bouquet A, Williams LE. Cambridge: University Press; 1992:112-146.

7. Ojeda H, Deloire A, Carbonneau A, Ageorges A, Romieu C: Berry development of grapevines: relations between the growth of berries and their DNA content indicate cell multiplication and enlargement. Vitis 1999, 38:145-150.

8. Friend AP, Trought MCT, Creasy GL: The influence of seed weight on the development and growth of berries and live green ovaries in Vitis vinifera L. cvs. Pinot Noir and Cabernet Sauvignon. Aust I Grape Wine Res 2009, 15:166-174.

9. Ollat N, Diakou-Verdin P, Carde JP, Barrieu F, Gaudillère JP, Moing A: Grape berry development: a review. J Int Sci Vigne Vin 2002, 36:109-131.

10. Chervin C, El-Kereamy A, Roustan JP, Latche A, Lamon J, Bouzayen M: Ethylene seems required for the berry development and ripening in grape, a non-climacteric fruit. Plant Sci 2004, 167:1301-1305.

11. Chervin C, Tira-umphon A, Terrier N, Zouine M, Severac D, Roustan JP: Stimulation of the grape berry expansion by ethylene and effects on related gene transcripts, over the ripening phase. Physiol Plant 2008, 134:534-546.

12. Davies C, Boss PK, Robinson SP: Treatment of grape berries, a nonclimacteric fruit with a synthetic auxin, retards ripening and alters the expression of developmentally regulated genes. Plant Physiol 1997, 115:1155-1161.

13. Scienza A, Miravallec C, Fregoni M: Relationship between seed number gibberellin and abcissic acid levels and ripening in Cabernet Sauvignon grape berries. Vitis 1978, 17:361-368.

14. Moreno D, Berli F, Piccoli P, Bottini R: Gibberellins and abscisic acid promote carbon allocation in roots and berries of grapevines. $J$ Plant Growth Regul 2011, 30:220-228.

15. Cadot Y, Miñana-Castelló MT, Chevalier M: Anatomical, histological, and histochemical changes in grape seeds from Vitis vinifera L. CV cabernet franc during fruit development. J Agric Food Chem 2006, 54:9206-9215

16. Ledbetter CA, Ramming DW: Seedlessness in grapes. Horticultural Review 1989, 11:159-184.

17. Striem MJ, Ben-Hayyim G, Spiegel-Roy P: Identifying molecular genetic markers associated with seedlessness in grape. J Am Soc Hortic Sci 1996, 121:758-763.

18. Staudt G, Schneider W, Leidel J: Phases of berry growth in Vitis vinifera. Ann Bot 1986, 58:789-800.
19. Boselli M, Volpe B, Di Vaio C: Effect of seed number per berry on mineral composition of grapevine (Vitis vinifera L.) berries. J Hortic Sci 1995, 70:509-515

20. Ebadi A, May P, Coombe BG: Effect of short-term temperature and shading on fruit-set, seed and berry development in model vines of $V$. vinifera, cvs Chardonnay and Shiraz. Aust I Grape Wine Res 1996, 2:2-9.

21. May P: From bud to berry, with special reference to inflorescence and bunch morphology in Vitis vinifera L. Aust J Grape Wine Res 2000, 6:82-98.

22. Petrie PR, Trought MCT, Howell GS: Fruit composition and ripening of Pinot Noir (Vitis vinifera L.) in relation to leaf area. Aust I Grape Wine Res 2000, 6:46-51.

23. Walker RR, Deidre HB, Clingeleffer PR, Kerridge GH, Rühl EH, Nicholas PR: Shiraz berry size in relation to seed number and implications for juice and wine composition. Aust J Grape Wine Res 2005, 11:2-8.

24. Wagner R, Antcliff J: A study of sexual progenies of Bicane $x$ Sultanina (Vitis vinifera L.): evidence for genetic differences between Sultana clones in berry weight. In Proceedings of the IIIrd Symposium on Grape Genetics and Breeding; 1980:65-77.

25. Doligez A, Bouquet A, Danglot Y, Lahogue F, Riaz S, Meredith CP, Edwards K, This P: Genetic mapping of grapevine (Vitis vinifera L.) applied to the detection of QTLs for seedlessness and berry weight. Theor Appl Genet 2002, 105:780-795.

26. Fanizza G, Lamaj F, Costantini L, Chaabane R: QTL analysis for fruit yield components in table grapes (Vitis vinifera). Theor Appl Genet 2005, 111:658-664.

27. Cabezas JA, Cervera MT, Ruiz-Garcia L, Carreño J, Martinez-Zapater JM: A genetic analysis of seed and berry weight in grapevine. Genome 2006, 49:1572-1585

28. Mejia N, Gebauer M, Muñoz L, Hewstone N, Muñoz C, Hinrichsen P: Identification of QTLs for seedlessness, berry size, and ripening date in a seedless $x$ seedless table grape progeny. Am J Enol Vitic 2007, 58:499-507.

29. Costantini L, Battilana J, Lamaj F, Fanizza G, Grando MS: Berry and phenology-related traits in grapevine (Vitis vinifera L.): from quantitative trait loci to underlying genes. BMC Plant Biol 2008, 8:38.

30. Meijia N, Soto B, Guerrero M, Casanueva X, Houel C, De los Angeles Miccono M, Ramos R, Le Cunff L, Boursiquot JM, Hinrichsen P, AdamBlondon AF: Molecular, genetic and transcriptional evidence for a role of VvaGL11 in stenospermocarpic seedlessness in grapevine. BMC Plant Biol 2011, 11:57.

31. Wei $X$, Sykes SR, Clingeleffer PR: An investigation to estimate genetic parameters in CSIRO's table grape breeding program 2: quality characteristics. Euphytica 2002, 128:343-351.

32. Ebadi A, Moghadam JE, Fatahi R: Evaluation of 22 populations achieved from controlled crossing between some seeded $x$ seedless grapevine cultivars. Sci Hortic 2009, 119:371-376.

33. Wagner R: Study of phenotypic variation by analysing data gathered together by O.I.V. on new varieties of table grapes. In Proceedings of the Vth Symposium on Grape Genetics and Breeding. ; 1989:178-186.

34. Coombe BG: Relationship of growth and development to changes in sugars, auxins, and gibberellins in fruit of seeded and seedless varieties of Vitis vinifera. Plant Physiol 1960, 35:241-250.

35. Perez FJ, Viani C, Retamales J: Bioactive gibberellins in seeded and seedless grapes: identification and changes in content during berry development. Am J Enol Vitic 2000, 51:315-318.

36. Weaver RJ, Pool RM: Relation of seededness and ringing to gibberellin-like activity in berries of Vitis vinifera. Plant Physiol 1965, 40:770-776.

37. Considine JA, Coombe BG: The interaction of gibberellic acid and 2-(chloroethyl) trimethyl ammonium chloride on fruit cluster development in Vitis vinifera L. (m. dt. Zus.). Vitis 1972, 11:108-123.

38. Eibach R: Investigations about the influence of some physiological and phenological characteristics on quality and their heredity. In Proceedings of the Vth Symposium on Grape Genetics and Breding. ; 1989:149-158.

39. Daulta BS, Bakhshi JC, Chandra S: Evaluation of vinifera varieties for genotypic and phenotypic variability. Indian J Hortic 1972, 29:150-157.

40. Golodriga PI, Trochine LP: Héritabilité des caractères quantitatifs chez la vigne. In Proceedings of the IInd Symposium on Grape Genetics and Breeding; 1978:113-117.

41. Firoozabady E, Olmo HP: Heritability and correlation studies of certain quantitative traits in table grapes, Vitis spp. Vitis 1987, 26:132-146.

42. Singh R, Jalikop SH: Studies on variability in grape. Indian J Hortic 1986, 43:207-215 
43. Fischer BM, Salakhutdinov I, Akkurt M, Eibach R, Edwards KJ, Töpfer R, Zyprian EM: Quantitative trait locus analysis of fungal disease resistance factors on a molecular map of grapevine. Theor Appl Genet 2004, 108:501-515.

44. Houel C, Bounon R, Chaïb J, Guichard C, Péros JP, Bacilieri R, Dereeper A, Canaguier A, Lacombe T, N'Diaye A, Le Paslier MC, Vernerey MS, Coriton O, Brunel D, This P, Torregrosa L, Adam-Blondon AF: Patterns of sequence polymorphism in the fleshless berry locus in cultivated and wild Vitis vinifera accessions. BMC Plant Biol 2010, 10:284.

45. Houel C: Caractérisation de la variation phénotypique de la taille de la baie chez la vigne Vitis vinifera L. et approches de génétique d'association et recherche de traces de sélection pour ce caractère. PhD thesis. Université d'Evry Val d'Essonne, France, Ecole doctorale des Génomes aux Organismes; 2011.

46. Bouquet $A$, Danglot $Y$ : Inheritance of seedlessness in grapevine (Vitis vinifera L.). Vitis 1996, 35:35-42.

47. Doligez A, Audiot E, Baumes R, This P: QTLs for muscat flavor and monoterpenic odorant content in grapevine (Vitis vinifera L.). Mol Breed 2006, 18:109-125.

48. Doligez A, Bertrand Y, Dias S, Grolier M, Ballester JF, Bouquet A, This P: QTLs for fertility in table grape (Vitis vinifera L.). Tree Genet Genomes 2010, 6:413-422.

49. Huang YF, Doligez A, Fournier-Level A, Le Cunff L, Bertrand Y, Canaguier A, Morel C, Miralles V, Veran F, Souquet JM, Cheynier V, Terrier N, This P: Dissecting genetic architecture of grape proanthocyanidin composition through quantitative trait locus mapping. BMC Plant Biol 2012, 12:30.

50. R Development Core Team: $R$ : a language and environment for statistical computing. Vienna, Austria: R Foundation for Statistical Computing; 2011. URL http://www.R-project.org/. ISBN ISBN 3-900051-07-0.

51. Royston P: Remark AS R94: a remark on algorithm AS 181: the W test for normality. App/ Stat 1995, 44:547-551.

52. Wang S, Basten CJ, Zeng ZB: Windows QTL Cartographer 2.0. User Manual. Department of Statistics, North Carolina State University; 2004 http://statgen.ncsu.edu/qtlcart/WinQTLCart.pdf.

53. Basten CJ, Weir BS, Zeng ZB: Zmap-a QTL cartographer. In Proceedings of the 5th World Congress on Genetics Applied to Livestock Production: Computing Strategies and Software. Edited by Smith C, Gavora JS, Benkel B, Chesnais J, Fairfull W, Gibson JP, Kennedy BW, Burnside EB. Guelph, Ontario, Canada: Organizing Committee of the 5th World Congress on Genetics Applied to Livestock Production; 1994:65-66.

54. Basten CJ, Weir BS, Zeng ZB: QTL Cartographer, Version 1.17: a Reference Manual and Tutorial for QTL Mapping. Raleigh, NC: Department of Statistics, North Carolina State University; 2003

55. Van Ooijen JW, Maliepaard C: MapQTL ${ }^{\mathrm{TM}}$ version 3.0: Software for the calculation of QTL positions on genetic maps. CPRO-DLO; 1996. http:// www.kyazma.nl/index.php/mc.MapQTL/.

56. Segura V, Denancé C, Durel C, Costes E: Wide range QTL analysis for complex architectural traits in a 1-year-old apple progeny. Genome 2007, 50:159-171.

57. De Franceschi P, Stegmeir T, Cabrera A, van der Knaap E, Rosyara UR, Sebolt AM, Dondini L, Dirlewanger E, Quero-Garcia J, Campoy JA, lezzoni AF: Cell number regulator genes in Prunus provide candidate genes for the control of fruit size in sweet and sour cherry. Mol Breeding 2013, 32:311-326.

58. Chakrabarti M, Zhang N, Sauvage C, Muños S, Blanca J, Cañizares J, Diez MJ, Schneider R, Mazourek M, McClead J, Causse M, van der Knaap E: A cytochrome $\mathrm{P} 450$ regulates a domestication trait in cultivated tomato. P Natl Acad Sci USA 2013, 110:17125-17130.

59. Price AH: Believe it or not, QTLs are accurate! Trends Plant Sci 2006 $11: 213-216$

60. Guo M, Rupe MA, Dieter JA, Zou JJ, Spielbauer D, Duncan KE, Howard RJ, Hou ZL, Simmons CR: Cell number regulator1 affects plant and organ size in maize: implications for crop yield enhancement and heterosis. Plant Cell 2010, 22:1057-1073.

61. Poupin M, Federici F, Medina C, Matus J, Timmermann T, Arce-Johnson P. Isolation of the three grape sub-lineages of B-class MADS-box TM6, PISTILLATA and APETALA3 genes which are differentially expressed during flower and fruit development. Gene 2007, 404:10-24.

62. Fernandez L, Torregrosa L, Terrier N, Sreekantan L, Grimplet J, Davies C, Thomas MR, Romieu C, Ageorges A: Identification of genes associated with flesh morphogenesis during grapevine fruit development. Plant Mol Biol 2007, 63:307-323.
63. Schlosser J, Olsson N, Weis M, Reid K, Peng F, Lund S, Bowen P: Cellular expansion and gene expression in the developing grape (Vitis vinifera L.). Protoplasma 2008, 232:255-265.

64. Lijavetzky D, Carbonell-Bejerano P, Grimplet J, Bravo G, Flores P, Fenoll J, Hellin P, Oliveros J, Martinez-Zapater J: Berry flesh and skin ripening features in Vitis vinifera as assessed by transcriptional profiling. PLOS ONE 2012, 7:e39547

65. Guillaumie S, Fouquet R, Kappel C, Camps C, Terrier N, Moncomble D, Dunlevy J, Davies C, Boss P, Delrot S: Transcriptional analysis of late ripening stages of grapevine berry. BMC Plant Biol 2011, 11:165.

66. Nunan KJ, Davies C, Robinson SP, Fincher GB: Expression patterns of cell wall-modifying enzymes during grape berry development. Planta 2001, 214:257-264.

67. Boss PK, Sensi E, Hua C, Davies C, Thomas MR: Cloning and characterisation of grapevine (Vitis vinifera L.) MADS-box genes expressed during inflorescence and berry development. Plant Sci 2002, 162:887-895

68. Yang G, Cao X, Fang J, Huang Z, Tao J, Wang C: Sub-cellular localization and expression analysis of genes involved in grapevine floral development. Sci Agric Sin 2011, 44:641-650.

69. Frary A, Nesbitt TC, Frary A, Grandillo S, van der Knaap E, Cong B, Liu J, Meller J, Elber R, Alpert KB, Tanksley SD: fw2.2: a quantitative trait locus key to the evolution of tomato fruit size. Science 2000, 289:85-88.

70. Segura V, Durel C, Costes E: Dissecting apple tree architecture into genetic, ontogenetic and environmental effects: QTL mapping. Tree Genet Genomes 2009, 5:165-179.

71. Fournier-Level A, Le Cunff L, Gomez C, Doligez A, Ageorges A, Roux C, Bertrand Y, Souquet JM, Cheynier V, This P: Quantitative genetic bases of anthocyanin variation in grape (Vitis vinifera L. ssp. sativa) berry: a quantitative trait locus to quantitative trait nucleotide integrated study. Genetics 2009, 183:1127-1139.

72. Duchene E, Butterlin G, Dumas V, Merdinoglu D: Towards the adaptation of grapevine varieties to climate change: QTLs and candidate genes for developmental stages. Theor App/ Genet 2012, 124:623-635.

73. Broman KW, Wu H, Sen Ś, Churchill GA: R/qtl: QTL mapping in experimental crosses. Bioinformatics 2003, 19:889-890.

74. Dalbo MA, Ye GN, Weeden NF, Steinkellner H, Sefc KM, Reisch BI: A gene controlling sex in grapevines placed on a molecular marker-based genetic map. Genome 2000, 43:333-340.

75. Lowe KM, Walker MA: Genetic linkage map of the interspecific grape rootstock cross Ramsey (Vitis champinii) x Riparia Gloire (Vitis riparia). Theor Appl Genet 2006, 112:1582-1592.

76. Riaz S, Krivanek AF, Xu K, Walker MA: Refined mapping of Pierce's disease resistance locus, $P d R 1$, and sex on an extended genetic map of Vitis rupestris x V. arizonica. Theor Appl Genet 2006, 113:1317-1329.

77. Marguerit E, Boury C, Manicki A, Donnart M, Butterlin G, Nemorin A, Wiedemann-Merdinoglu S, Merdinoglu D, Ollat N, Decrooca S: Genetic dissection of sex determinism, inflorescence morphology and downy mildew resistance in grapevine. Theor App/ Genet 2009, 118:1261-1278.

78. Appazova AO: Induction of parthenocarpy in grape. Doklady TSKhA 1977, 231:102-105.

79. Uzun I, Agaoglu S, Soylemezoglu G: Ampelographic characteristics and isozymic analysis of wild grapevines ( Vitis vinifera ssp. silvestris Gmel.) in southwestern Turkey. In Proceedings of the International symposium on in situ conservation of plant genetic diversity, 4-8 November 1996; Antalya, Turkey. Edited by Zencirci N, Kaya Z, Anikster Y, Adams WT. Ankara; Turkey: Central Research Institute for Field Crops; 1998:163-169.

80. Hofacker W: Investigations into seed weight and seed number of grapevine and their influence on berry weight. Mitt Klosterneuburg Rebe Wein Obstb Fruchteverwert 2003, 53:235-242

81. Myles S, Boyko AR, Owens CL, Brown PJ, Grassi F, Aradhya MK, Prins B, Reynolds A, Chia JM, Ware D, Bustamante CD, Buckler ES: Genetic structure and domestication history of the grape. Proc Natl Acad Sci USA 2011 108:3530-3535.

82. Grassi F, Labra M, Imazio S, Spada A, Sgorbati S, Scienza A, Sala F: Evidence of a secondary grapevine domestication centre detected by SSR analysis. Theor Appl Genet 2003, 107:1315-1320.

83. Riahi L, Laucou V, Le Cunff L, Zoghlami N, Boursiquot J, Lacombe T, El-Heit K, Mliki A, This P: Highly polymorphic nSSR markers: a useful tool to assess origin of North African cultivars and to provide additional proofs of secondary grapevine domestication events. Sci Hortic 2012, 141:53-60. 
84. Gillapsy G, Ben-David H, Gruissem W: Fruits: a developmental perspective. Plant Cell 1993, 5:1439-1451.

85. Robinson SP, Davies C: Molecular biology of grape berry ripening. Aust J Grape Wine Res 2000, 6:175-188.

86. Espinoza A, Contreras D, Orellana M, Perez R, Aguirre C, Castro A, Riquelme A, Fichet $T$, Pinto $M$, Hinrichsen P: Modulation by gibberellic acid of aquaporin genes expression during berry development of grapevine (Vitis vinifera L.). Acta Hort 2009:355-362.

87. Shelden M, Howitt S, Kaiser B, Tyerman S: Identification and functional characterisation of aquaporins in the grapevine, Vitis vinifera. Funct Plant Biol 2009, 36:1065-1078

88. Zenoni S, Ferrarini A, Giacomelli E, Xumerle L, Fasoli M, Malerba G, Bellin D, Pezzotti M, Delledonne M: Characterization of transcriptional complexity during berry development in Vitis vinifera using RNA-seq. Plant Physiol 2010, 152:1787-1795.

89. Kaps ML, Cahoon GA: Growth and fruiting of container-grown Seyval blanc grapevines modified by changes in crop level, leaf number and position, and light exposure. Am J Enol Vitic 1992, 43:191-199.

90. Peng F, Reid K, Liao N, Schlosser J, Lijavetzky D, Holt R, Martinez Zapater J, Jones S, Marra M, Bohlmann J, Lund S: Generation of ESTs in Vitis vinifera wine grape (Cabernet Sauvignon) and table grape (Muscat Hamburg) and discovery of new candidate genes with potential roles in berry development. Gene 2007, 402:40-50.

91. Boss PK, Thomas MR: Association of dwarfism and floral induction with a grape 'green revolution' mutation. Nature 2002, 416:847-850.

92. Diaz-Riquelme J, Lijavetzky D, Martinez-Zapater JM, Carmona MJ: Genome-wide analysis of MIKC C-type MADS box genes in grapevine. Plant Physio/ 2009, 149:354-369.

93. Davies $C$, Wolf $T$, Robinson $\mathrm{S}$ : Three putative sucrose transporters are differentially expressed in grapevine tissues. Plant Sci (Limerick) 1999 147:93-100

94. Nicolas P, Lecourieux D, Gomès E, Delrot S, Lecourieux F: The grape berryspecific basic helix-loop-helix transcription factor VVCEB1 affects cell size. J Exp Bot 2013, 64:991-1003.

95. Ito T, Meyerowitz EM: Overexpression of a gene encoding a cytochrome p450, CYP78A9, induces large and seedless fruit in Arabidopsis. Plant Cell 2000, 12:1541-1550.

96. Suzuki M, Kamide Y, Nagata N, Seki H, Ohyama K, Kato H, Masuda K, Sato S, Kato T, Tabata S, Yoshida S, Muranaka T: Loss of function of 3-hydroxy-3methylglutaryl coenzyme A reductase 1 (HMG1) in Arabidopsis leads to dwarfing, early senescence and male sterility, and reduced sterol levels. Plant J 2004, 37:750-761.

97. Wang XC, Ren GH, Fang JG, Lia Y, Liu H, Wu WM, Zhao MZ: Cloning, subcellular localization and expression analysis of genes related to the synthesis of gibberellin from grapevine. Sci Agric Sin 2012, 45:2224-2231.

98. Davies C, Robinson SP: Differential screening indicates a dramatic change in $\mathrm{mRNA}$ profiles during grape berry ripening: cloning and characterization of cDNAs encoding putative cell wall and stress response proteins. Plant Physiol 2000, 122:803-812.

99. Shangguan L, Han J, Fang J, Wang X, Leng X: Identification of grape (Vitis vinifera $\mathrm{L}$.) genes from EST sequences responding to exogenous gibberellins treatment. J Agric Biotech 2012, 20:135-145.

100. Silverstone AL, Tseng TS, Swain SM, Dill A, Jeong SY, Olszewski NE, Sun TP: Functional analysis of SPINDLY in gibberellin signaling in Arabidopsis. Plant Physiol 2007, 143:987-1000.

101. Nishimura T, Wada T, Yamamoto KT, Okada K: The Arabidopsis STV1 protein, responsible for translation reinitiation, is required for auxinmediated gynoecium patterning. Plant Cell 2005, 17:2940-2953.

102. Boss PK, Vivier M, Matsumoto S, Dry IB, Thomas MR: A cDNA from grapevine ( Vitis vinifera L.), which shows homology to AGAMOUS and SHATTERPROOF, is not only expressed in flowers but also throughout berry development. Plant Mol Biol 2001, 45:541-553.

103. Ishimaru M, Smith D, Gross K, Kobayashi S: Expression of three expansin genes during development and maturation of Kyoho grape berries. J Plant Physiol 2007, 164:1675-1682.

104. Hanania U, Velcheva M, Sahar N, Flaishman M, Or E, Degani O, Perl A: The ubiquitin extension protein S27a is differentially expressed in developing flower organs of Thompson seedless versus Thompson seeded grape isogenic clones. Plant Cell Rep 2009, 28:1033-1042.
105. Fillion L, Ageorges A, Picaud S, Coutos-Thevenot P, Lemoine R, Romieu C, Delrot $\mathrm{S}$ : Cloning and expression of a hexose transporter gene expressed during the ripening of grape berry. Plant Physiol 1999, 120:1083-1093.

106. Wang S, Yu Y, Zhang C, Xu W, Wang Y: Molecular cloning and characterization of a novel gene encoding an EF-hand calcium-binding protein related to fruit seedlessness of grapevine. Sci Hortic 2011, 130:708-714.

doi:10.1186/1471-2229-13-217

Cite this article as: Doligez et al:: New stable QTLs for berry weight do not colocalize with QTLs for seed traits in cultivated grapevine (Vitis vinifera L.). BMC Plant Biology 2013 13:217.

\section{Submit your next manuscript to BioMed Central and take full advantage of:}

- Convenient online submission

- Thorough peer review

- No space constraints or color figure charges

- Immediate publication on acceptance

- Inclusion in PubMed, CAS, Scopus and Google Scholar

- Research which is freely available for redistribution 